\title{
Convergence of the self-energy in a relativistic chiral quark model: excited Nucleon and $\Delta$ sector
}

\author{
E. M. Tursunov \\ Institute of Nuclear Physics, Uzbekistan Academy of Sciences, 100214, Ulugbek, Tashkent, Uzbekistan
}

(Dated: April 22, 2022)

\begin{abstract}
A convergence of the valence quark self-energies in the $1 S, 2 S, 1 P_{1 / 2}, 1 P_{3 / 2}$ orbits induced by pion and gluon field configurations, is shown in the frame of a relativistic chiral quark model. It is shown that in order to reach a convergence, one needs to include contribution of the intermediate quark and anti-quark states with the total momentum up to $j=25 / 2$. It is argued that a restriction to the lowest mode when estimating the self-energy is not good approximation.

PACS numbers: 11.10.Ef, 12.39.Fe, 12.39.Ki, 13.40.Em,13.40.Gp, 14.20.Dh
\end{abstract}

\section{INTRODUCTION}

Current programs of the world leading facilities such as JLAB and MAMI, aims to study the properties of the excited $N^{*}$ and $\Delta^{*}$ resonances (see Ref. [1-4]). New data obtained in these experiments yield valuable information on effective degrees of freedom in the low-energy regime of the QCD.

In spite of many efforts, there still exist a number of serious problems in the baryon spectroscopy. First of them is the problem of missing resonances, which were predicted by the Constituent Quark Models (CQM) [5-7], but are still being not observed at the current experiments. Additionally, the structure of the Roper resonance is not yet well understood. It is not yet clear, what is the mechanism which lowers the Roper's mass.

An opposite picture on the baryon structure is based on the relativistic description. Although the CQMs are well adapted for the calculations of the ground and excited baryon spectrum, arguments against the CQM are very serious: a valence quark inside the baryon moves with a velocity, close to the speed of the light. Another fact is that a single pion or gluon can be emitted by a valence quark and be absorbed by the same quark (through self-energy diagrams). After emitting a single boson, the intermediate quark can excite to the quark or antiquark states, thus yielding the contribution of the sea-quarks to the baryon mass-spectrum. Of course, such diagrams can not be involved in the CQMs. An alternative relativistic Cloudy Bag Model [8] was not extended to the excited Nucleon and $\Delta$ sector, though it could explain the proton spin problem [9].

In Ref. 10, 11] we have developed a relativistic chiral quark model 12 14 for the excited $N^{*}$ and $\Delta^{*}$ spectroscopy. The model includes one-gluon and one-pion loop diagrams contributions and reproduces the main properties of the lowest excitation spectrum of the Nucleon and $\Delta$. It was shown that relativistic one-meson and one-gluon exchange forces generate spin $0,1,2$, etc. operators, which couple the lower and the upper components of the two interacting valence quarks. The only contribution to the ground state nucleon and $\Delta$ comes from the spin 1 operators, which correspond to the exchanged pion or gluon in the $\mathrm{l}=1$ orbit. This result can explain naturally, why the $\Delta\left(3 / 2^{+}\right)(1232)$ resonance appears in the $P$-wave of the $\pi N$ scattering data, which can not be understood in the frame of the Constituent Quark Models.

The aim of present paper is to show the convergence of the valence quark self-energy diagrams induced by the pion and gluon field configurations. A convergence of the self-energy induced by pion fields in the present model has been demonstrated for the valence quarks in the ground state [13, 14]. However, an extension of the model to the excited $N^{*}$ and $\Delta^{*}$ requires to study the behavior of the self-energy for the excited valence quark states. As it is well known [15], the convergence of the self-energy is a serious problem for the bag models due-to the sharp surface quark-pion coupling. It will be shown in our model, that to reach convergence, one needs to include the contribution of the intermediate quark and antiquark states with a total momentum up to $j=25 / 2$. This is why a restriction to the lowest modes used in many relativistic calculations is not a good approximation [16].

The relativistic quark model is based on an effective chiral Lagrangian describing quarks as relativistic fermions moving in a confining static potential. The potential is described by a Lorentz scalar and the time component of a vector potential, where the latter term is responsible for short-range fluctuations of the gluon field configurations [17]. The model potential defines unperturbed wave functions of the quarks which are subsequently used in the calculations of baryon properties. The baryons are considered as bound states of valence quarks, surrounded by a pion cloud as required by the chiral symmetry and by gluons. Interaction of quarks with a pion is introduced on the basis of the linearized $\sigma$-model [13, 14, 18]. The quark-gluon interaction is introduced on the field-theoretical basis as prescribed by QCD. Calculations are performed perturbatively to second order in the quark-pion and quark-gluon interaction. All calculations are performed at one loop or at order of accuracy $o\left(1 / f_{\pi}^{2}, \alpha_{s}\right)$.

In the following we first give the basic formalism of our approach, then present the numerical results. 


\section{MODEL}

The effective Lagrangian of our model $\mathcal{L}(x)$ contains the quark core part $\mathcal{L}_{Q}(x)$ the quark-pion $\mathcal{L}_{I}^{(q \pi)}(x)$ and the quark-gluon $\mathcal{L}_{I}^{(q g)}(x)$ interaction parts, and the kinetic parts for the pion $\mathcal{L}_{\pi}(x)$ and gluon $\mathcal{L}_{g}(x)$ :

$$
\begin{array}{r}
\mathcal{L}(x)=\mathcal{L}_{Q}(x)+\mathcal{L}_{I}^{(q \pi)}(x)+\mathcal{L}_{I}^{(q g)}(x)+\mathcal{L}_{\pi}(x)+\mathcal{L}_{g}(x) \\
=\bar{\psi}(x)\left[i \not \partial-S(r)-\gamma^{0} V(r)\right] \psi(x)-1 / f_{\pi} \bar{\psi}\left[S(r) i \gamma^{5} \tau^{i} \phi_{i}\right] \psi- \\
-g_{s} \bar{\psi} A_{\mu}^{a} \gamma^{\mu} \frac{\lambda^{a}}{2} \psi+\frac{1}{2}\left(\partial_{\mu} \phi_{i}\right)^{2}-\frac{1}{4} G_{\mu \nu}^{a} G_{a}^{\mu \nu} .
\end{array}
$$

Here, $\psi(x), \phi_{i}, i=1,2,3$ and $A_{\mu}^{a}$ are the quark, the pion and the gluon fields, respectively. The matrices $\tau^{i}(i=1,2,3)$ and $\lambda^{a}(a=1, \ldots, 8)$ are the isospin and color matrices, correspondingly. The pion decay constant $f_{\pi}=93 \mathrm{MeV}$. The scalar part of the static confinement potential is given by

$$
S(r)=c r+m
$$

where $\mathrm{c}$ and $\mathrm{m}$ are constants.

At short distances, transverse fluctuations of the string are dominating [17], with some indication that they transform like the time component of the Lorentz vector. They are given by a Coulomb type vector potential as

$$
V(r)=-\alpha / r
$$

where $\alpha$ is approximated by a constant. The quark fields are obtained from solving the Dirac equation with the corresponding scalar plus vector potentials

$$
\left[i \gamma^{\mu} \partial_{\mu}-S(r)-\gamma^{0} V(r)\right] \psi(x)=0
$$

The respective positive and negative energy eigenstates as solutions to the Dirac equation with a spherically symmetric mean field, are given in a general form as

$$
\begin{aligned}
& u_{\alpha}(x)=\left(\begin{array}{c}
g_{N \kappa}^{+}(r) \\
-i f_{N \kappa}^{+}(r) \vec{\sigma} \hat{\vec{x}}
\end{array}\right) \mathcal{Y}_{\kappa}^{m_{j}}(\hat{\vec{x}}) \chi_{m_{t}} \chi_{m_{c}} \exp \left(-i E_{\alpha} t\right) \\
& v_{\beta}(x)=\left(\begin{array}{c}
g_{N \kappa}^{-}(r) \\
-i f_{N \kappa}^{-}(r) \vec{\sigma} \hat{\vec{x}}
\end{array}\right) \mathcal{Y}_{\kappa}^{m_{j}}(\hat{\vec{x}}) \chi_{m_{t}} \chi_{m_{c}} \exp \left(+i E_{\beta} t\right)
\end{aligned}
$$

The quark and anti-quark eigenstates $u$ and $v$ are labeled by the radial, angular, azimuthal, isospin and color quantum numbers $N, \kappa, m_{j}, m_{t}$ and $m_{c}$, which are collectively denoted by $\alpha$ and $\beta$, respectively. The spin-angular part of the quark field operators

$$
\mathcal{Y}_{\kappa}^{m_{j}}(\hat{\vec{x}})=\left[Y_{l}(\hat{\vec{x}}) \otimes \chi_{1 / 2}\right]_{j m_{j}} j=|\kappa|-1 / 2 .
$$

The quark fields $\psi$ are expanded over the basis of positive and negative energy eigenstates as

$$
\psi(x)=\sum_{\alpha} u_{\alpha}(x) b_{\alpha}+\sum_{\beta} v_{\beta}(x) d_{\beta}^{\dagger}
$$

The expansion coefficients $b_{\alpha}$ and $d_{\beta}^{\dagger}$ are operators, which annihilate a quark and create an anti-quark in the orbits $\alpha$ and $\beta$, respectively.

The free pion field operator is expanded over plane wave solutions as

$$
\phi_{j}(x)=(2 \pi)^{-3 / 2} \int \frac{d^{3} k}{\left(2 \omega_{k}\right)^{1 / 2}}\left[a_{j \mathbf{k}} \exp (-i k x)+a_{j \mathbf{k}}^{\dagger} \exp (i k x)\right]
$$

with the usual destruction and creation operators $a_{j \mathbf{k}}$ and $a_{j \mathbf{k}}^{\dagger}$ respectively. The pion energy is defined as $\omega_{k}=\sqrt{k^{2}+m_{\pi}^{2}}$. The expansion of the free zero mass gluon field operators is of the same form. 
In denoting the three-quark vacuum state by $\mid 0>$, the corresponding noninteracting many-body quark Green's function (propagator) is given by the customary vacuum Feynman propagator for a binding potential [19]:

$$
i G\left(x, x^{\prime}\right)=i G^{F}\left(x, x^{\prime}\right)=<0\left|T\left\{\psi(x) \bar{\psi}\left(x^{\prime}\right)\right\}\right| 0>=\sum_{\alpha} u_{\alpha}(x) \bar{u}_{\alpha}\left(x^{\prime}\right) \theta\left(t-t^{\prime}\right)+\sum_{\beta} v_{\beta}(x) \bar{v}_{\beta}\left(x^{\prime}\right) \theta\left(t^{\prime}-t\right)
$$

Since the three-quark vacuum state $\mid 0>$ does not contain any pion or gluon, the pion and gluon Green's functions are given by the usual free Feynman propagator for a boson field:

$$
\begin{gathered}
i \Delta_{i j}\left(x-x^{\prime}\right)=<0\left|T\left\{\phi_{i}(x) \bar{\phi}_{j}\left(x^{\prime}\right)\right\}\right| 0>=i \delta_{i j} \int \frac{d^{4} k}{(2 \pi)^{4}} \frac{1}{k^{2}-m_{\pi}^{2}+i \epsilon} \exp \left[-i k\left(x-x^{\prime}\right)\right] \\
i \Delta_{a b}^{(\mu \nu)}\left(x-x^{\prime}\right)=<0\left|T\left\{A_{\mu}^{a}(x) A_{\nu}^{b}\left(x^{\prime}\right)\right\}\right| 0>=i \delta_{a b} g^{\mu \nu} \int \frac{d^{4} k}{(2 \pi)^{4}} \frac{1}{k^{2}+i \epsilon} \exp \left[-i k\left(x-x^{\prime}\right)\right],
\end{gathered}
$$

(in the Coulomb gauge), where we choose $g^{\mu \nu}=\delta_{\mu \nu} g^{\mu \mu}, g^{00}=-g^{11}=-g^{22}=-g^{33}=1$.

Using the effective Lagrangian and the time-ordered perturbation theory one can develop a calculation scheme for the lower excitation spectrum of the nucleon and delta. In the model the quark core result $\left(E_{Q}\right)$ is obtained by solving Eq.(4) for the single quark system numerically. Since we work in the independent particle model and limited with the lower excitation spectrum of the nucleon and Delta, the bare three-quark state of the $S U(2)$-flavor baryons corresponds to the structure $\left(1 S_{1 / 2}\right)^{2}(n l j)$ in the non-relativistic spectroscopic notation. The corresponding quark core energy is evaluated as the sum of single quark energies with:

$$
E_{Q}=2 E\left(1 S_{1 / 2}\right)+E(n l j)
$$

with an appropriate correction on the center of mass motion [1].

The second order perturbative corrections to the energy spectrum of the $\mathrm{SU}(2)$ baryons due to the pion field $\left(\Delta E^{(\pi)}\right)$ and the gluon fields $\left(\Delta E^{(g)}\right)$ are calculated on the basis of the Gell-Mann and Low theorem :

$$
\Delta E=<\Phi_{0}\left|\sum_{i=1}^{\infty} \frac{(-i)^{n}}{n !} \int i \delta\left(t_{1}\right) d^{4} x_{1} \ldots d^{4} x_{n} T\left[\mathcal{H}_{I}\left(x_{1}\right) \ldots \mathcal{H}_{I}\left(x_{n}\right)\right]\right| \Phi_{0}>_{c}
$$

with $n=2$, where the relevant quark-pion and quark-gluon interaction Hamiltonian densities are

$$
\begin{gathered}
\mathcal{H}_{I}^{(q \pi)}(x)=\frac{i}{f_{\pi}} \bar{\psi}(x) \gamma^{5} \vec{\tau} \vec{\phi}(x) S(r) \psi(x), \\
\mathcal{H}_{I}^{(q g)}(x)=g_{s} \bar{\psi}(x) A_{\mu}^{a}(x) \gamma^{\mu} \frac{\lambda^{a}}{2} \psi(x)
\end{gathered}
$$

The stationary bare three-quark state $\mid \Phi_{0}>$ is constructed from the vacuum state using the usual creation operators:

$$
\left|\Phi_{0}>_{\alpha \beta \gamma}=b_{\alpha}^{+} b_{\beta}^{+} b_{\gamma}^{+}\right| 0>
$$

where $\alpha, \beta$ and $\gamma$ represent the quantum numbers of the single quark states, which are coupled to the respective baryon configuration. The energy shift of Eq.(13) is evaluated up to second order in the quark-pion and quark-gluon interaction, and generates self-energy and exchange diagrams contributions.

The self-energy terms contain contribution both from intermediate quark $(E>0)$ and anti-quark $(E<0)$ states. These diagrams correspond to the case when a pion or gluon is emitted and absorbed by the same valence quark which is excited to the intermediate quark and anti-quark states. In this way one can estimate the contribution of the sea-quarks to the hadron spectrum that can not be done in non-relativistic quark models.

The pion part of the self energy term (pion cloud contribution) is evaluated as

$$
\Delta E_{s . e .}^{(\pi)}=-\frac{1}{2 f_{\pi}^{2}} \sum_{a=1}^{3} \sum_{\alpha^{\prime} \leq \alpha_{F}} \int \frac{d^{3} \vec{p}}{(2 \pi)^{3} p_{0}}\left\{\sum_{\alpha} \frac{V_{\alpha \alpha^{\prime}}^{a+}(\vec{p}) V_{\alpha \alpha^{\prime}}^{a}(\vec{p})}{E_{\alpha}-E_{\alpha^{\prime}}+p_{0}}-\sum_{\beta} \frac{V_{\beta \alpha^{\prime}}^{a+}(\vec{p}) V_{\beta \alpha^{\prime}}^{a}(\vec{p})}{E_{\beta}+E_{\alpha^{\prime}}+p_{0}}\right\}
$$


with $p_{0}^{2}=\vec{p}^{2}+m_{\pi}^{2}$. The $q-q-\pi$ transition form factors are defined as:

$$
\begin{aligned}
& V_{\alpha \alpha^{\prime}}^{a}(\vec{p})=\int d^{3} x \bar{u}_{\alpha}(\vec{x}) \Gamma^{a}(\vec{x}) u_{\alpha^{\prime}}(\vec{x}) e^{-i \vec{p} \vec{x}} \\
& V_{\beta \alpha^{\prime}}^{a}(\vec{p})=\int d^{3} x \bar{v}_{\beta}(\vec{x}) \Gamma^{a}(\vec{x}) u_{\alpha^{\prime}}(\vec{x}) e^{-i \vec{p} \vec{x}}
\end{aligned}
$$

The vertex function of the $\pi-q-q$ and $\pi-q-\bar{q}$ transition is

$$
\Gamma^{a}=S(r) \gamma^{5} \tau^{a} I_{c}
$$

where $I_{c}$ is the color unity matrix.

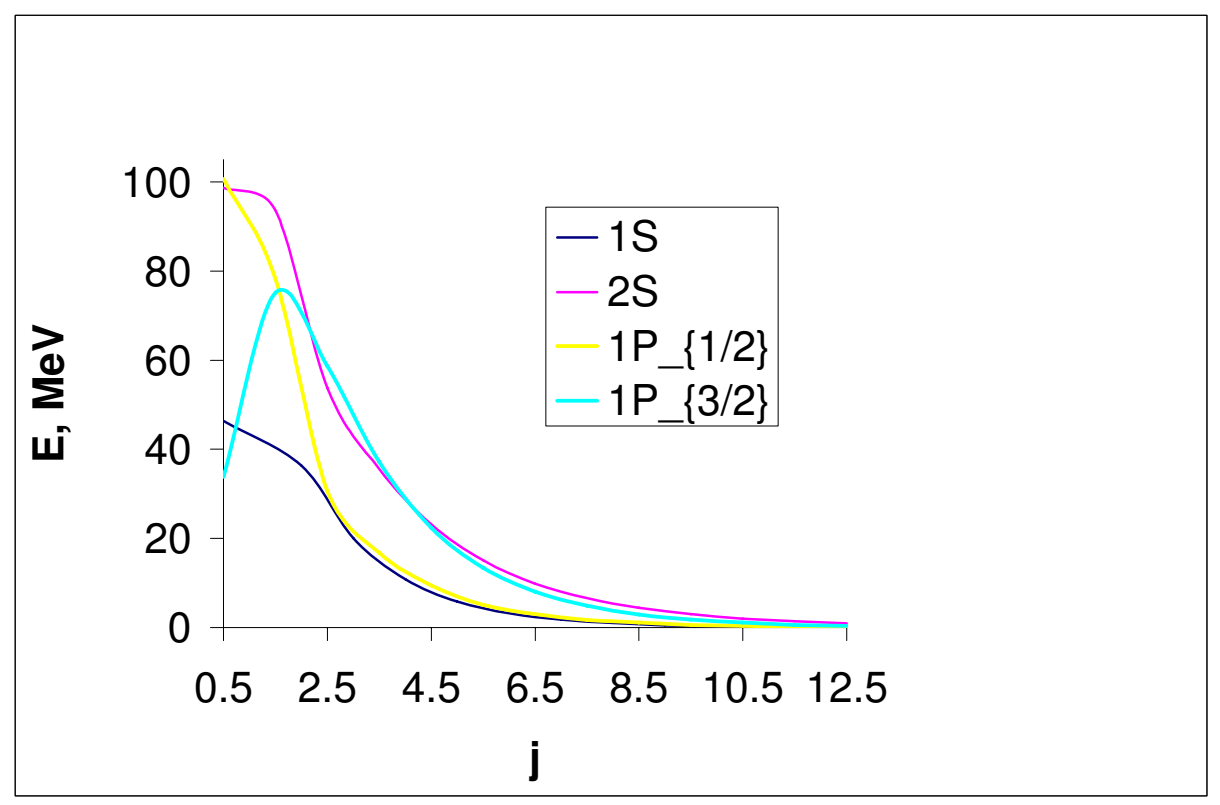

FIG. 1: Contributions self energy diagrams induced by $\pi-$ meson fields to the valence quark states in dependence on the total momentum of the intermediate quark (antiquark) states

The gluon part of the second order self-energy diagrams (gluon cloud) contribution is estimated in a similar fashion as

$$
\Delta E_{s . e .}^{(g)}=\frac{g_{s}^{2}}{2} \sum_{a} g_{\mu \nu} \sum_{\alpha^{\prime} \leq \alpha_{F}} \int \frac{d^{3} \vec{p}}{(2 \pi)^{3} p}\left\{\sum_{\alpha} \frac{V_{\alpha \alpha^{\prime}}^{a \mu+}(\vec{p}) V_{\alpha \alpha^{\prime}}^{a \nu}(\vec{p})}{E_{\alpha}-E_{\alpha^{\prime}}+p}-\sum_{\beta} \frac{V_{\beta \alpha^{\prime}}^{a \mu+}(\vec{p}) V_{\beta \alpha^{\prime}}^{a \nu}(\vec{p})}{E_{\beta}+E_{\alpha^{\prime}}+p}\right\}
$$

where the transition form factor is evaluated with the corresponding vertex matrix

$$
\Gamma_{\mu}^{a}=\gamma^{\mu} \frac{\lambda^{a}}{2} I_{t}
$$

with the isospin unity matrix $I_{t}$.

After evaluation of the transition form-factors and integration over angular variables, the self-energy terms induced by the both pion and gluon fields can be estimated in a proper way (see Ref. [11] for details). 


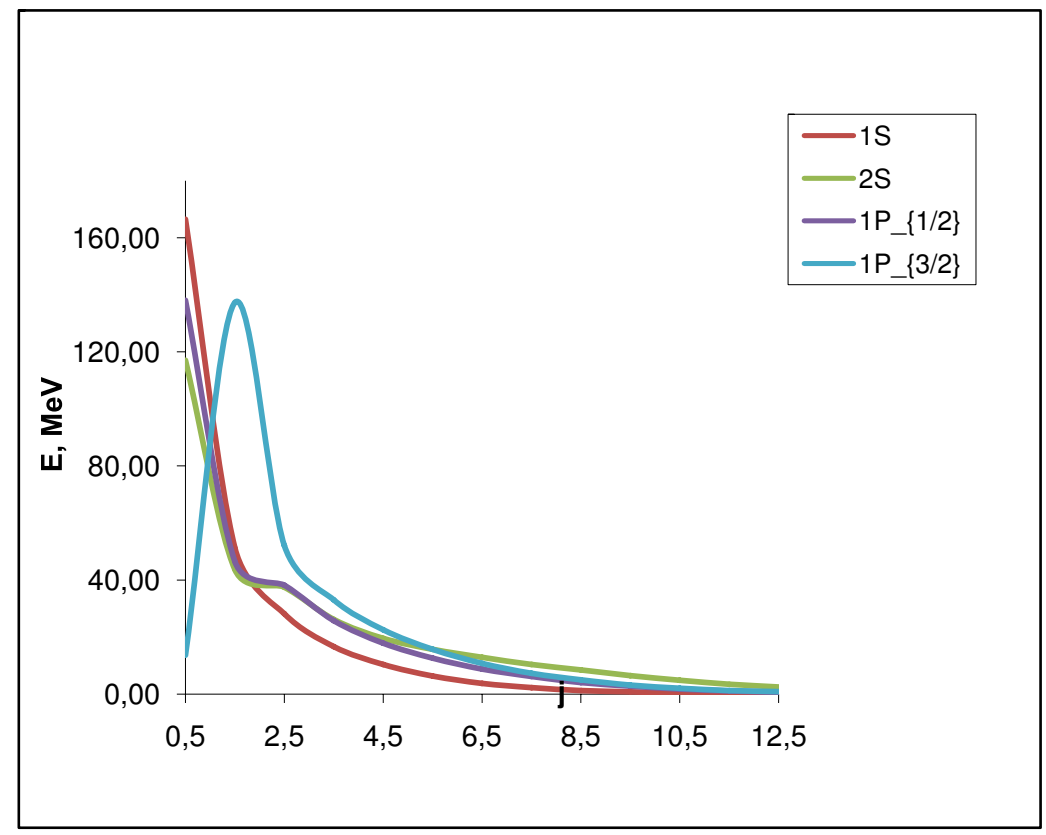

FIG. 2: Contributions self energy diagrams induced by by the color-electric components of the gluon fields

\section{NUMERICAL RESULTS}

In order to account for the finite size effect of the pion, we introduce an one-pion vertex regularization function in the momentum space, parameterized in the dipole form as

$$
F_{\pi}\left(p^{2}\right)=\frac{\Lambda_{\pi}^{2}-m_{\pi}^{2}}{\Lambda_{\pi}^{2}+p^{2}}
$$

From the flux-tube study [17] we fix $\alpha=0.26 \approx \pi / 12$. The strong coupling constant $g_{s}^{2}=4 \pi \alpha_{s}$ with the value $\alpha_{s}=0.65$. The parameters of the confining potential $\left(c=0.16 \mathrm{GeV}^{2}\right.$ and $\left.m=0.06 \mathrm{GeV}\right)$ are chosen to reproduce the correct axial charge $g_{A}$ of the proton (and the empirical pion-nucleon coupling constant $G_{\pi N N}^{2} / 4 \pi=14$ via the Goldberger-Treiman relation) and a normal value for the quark core RMS radius of the proton (see [13]). The pion-form factor $\Lambda_{\pi}=1.0 \mathrm{GeV}$ (see Model A in Ref [11]). It was shown in the Ref. [13] that the static properties of the proton with the correction on the center of mass motion $(\mathrm{CM})$ can be well reproduced with above parameters of the model.

In Fig. [1 we present the self-energy of the single valence quarks in the $1 S, 2 S, 1 P_{1 / 2}, 1 P_{3 / 2}$ orbits induced by the pion fields in the dependence on the total momentum of the intermediate quark and anti-quark states from $j=1 / 2$ up to $j=25 / 2$. One can note that the convergence is faster for the valence quark in the ground state $1 S$. The convergence also is reached for other valence quark states. We note here that a contribution of the intermediate quark states is negative, while intermediate anti-quark states yield larger and positive self-energy. As can be seen from the picture, the maximal contribution comes from the intermediate states with $j=1 / 2$ for the valence quarks except the $1 P_{3 / 2}$ orbit, which gets the most contribution from the intermediate quark and anti-quark states with the total momentum $j=3 / 2$. From this picture one can conclude that the sum of the self energy terms is limited and reasonable [11].

A very interesting behavior of the self-energy terms due-to color-electric field configurations can be found in the Fig.2. The self-energies of the $1 P_{1 / 2}$ and $2 S$ valence quarks, after rapid decreasing, have a plat around the interval $j=(3 / 2,5 / 2)$. Again, as in previous figures, we can see a reasonable convergence at $j=25 / 2$ for all valence quark states. 


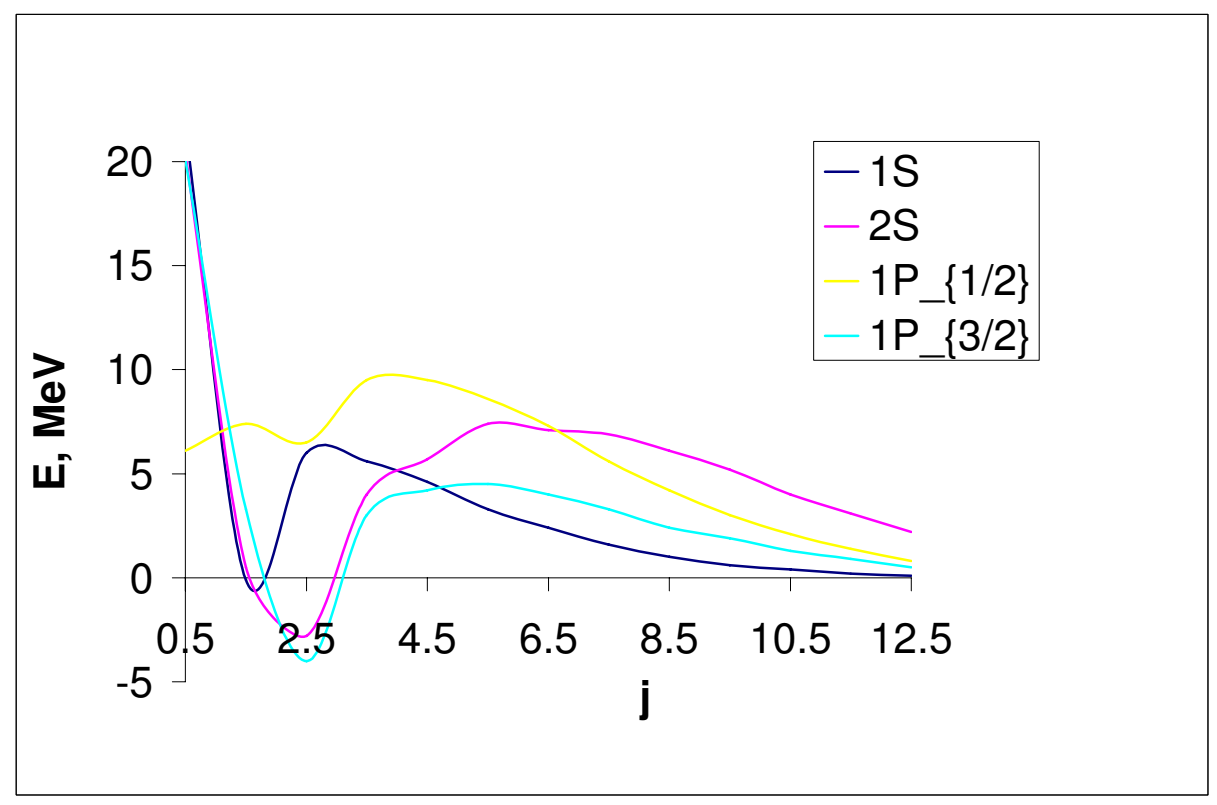

FIG. 3: Contributions self energy diagrams induced by by the color-magnetic components of the gluon fields

Finally, in Fig. 3 we show the behavior of the self-energy of the $1 S, 2 S, 1 P_{1 / 2}, 1 P_{3 / 2}$ valence quarks due-to colormagnetic fields. It's shape is quite different from that shown in the Fig. 1 and Fig. 2, For all the valence quark states, the self-energy has a minimum at small total momentum $j$ value. Even more, the intermediate quark states (with a negative self-energy contributions) dominate at this point over the intermediate anti-quark states for the valence quarks except the $1 P_{1 / 2}$ state. By further increasing the total momentum $j$ up to $25 / 2$, one can reach convergent results.

In Refs. 10, 11] it was shown, that the restriction of intermediate quark to the lowest mode is not a good approximation. As we see above, in order to reach convergent results, one needs to go up to large values of the total momentum $j$. The summary contribution of the pion fields to the $N(939)$ is about $200 \mathrm{MeV}$, while the gluon fields yield more than $500 \mathrm{MeV}$ shift with the strong coupling constant $\alpha_{s}=0.65$ mostly due-to color-electric field configurations. Contrary, the restriction to the intermediate ground state when estimating the self-energy (I=0) yields too small values for the nucleon energy (about $500 \mathrm{MeV}$ with the appropriate correction on the center of mass motion). Such estimations were obtained also for other lowest Nucleon and $\Delta$ states.

\section{SUMMARY AND CONCLUSIONS}

We have shown a convergence of the $1 S, 2 S, 1 P_{1 / 2}, 1 P_{3 / 2}$ valence quarks self-energies in the frame of a relativistic chiral quark model. It was shown that the convergent results for the self-energy due-to gluon and pion field configurations can be obtained with the inclusion of the intermediate quark and anti-quark states with the total momentum up to $j=25 / 2$. It is argued that the restriction to the lowest mode when estimating the self-energy is not a good approximation. 
Acknowledgements. Author thanks Th. Gutsche for useful discussions.

[1] Julia-Diaz B 2010 arXiv:1001.2909

[2] Kamano H, Julia-Diaz B, Lee T-S H, Matsuyama A, Sato T, 2009 arXiv:0909.1129

[3] Jankowiak A 2006 Eur. Phys. Jour. A28 Suppl. 1159

[4] Proc. of NSTAR 2007, 2008 Eur. Phys. Jour. A35 253-367

[5] Capstick S and Isgur N 1986 Phys. Rev. D34 2809

[6] Glozman L Y, Plessas W, Varga K and Wagenbrunn R F 1998 Phys. Rev. D58 094030

[7] Löring U, Kretzschmar K, Metsch B C and Petry H R 2001 Euro.Phys.Jour. A10 309 Löring U, Metsch B C and Petry H R 2001 Euro.Phys.Jour. A10 395

Löring U, Metsch B C and Petry H R 2001 Euro.Phys.Jour. A10 447

[8] Thomas A W, Theberge S and Miller G A 1980 Phys. Rev. D22 2838; 1981 Phys. Rev. D24 216

[9] Thomas A W 2008 Prog.Part.Nucl.Phys. 61219

Myhrer F and Thomas A W 2008 Phys.Lett. B663 302

[10] Tursunov E M 2005 J. Phys. G: Nucl. Part. Phys. 31617

[11] Tursunov E M 2009 J. Phys. G: Nucl. Part. Phys. 36095006

[12] Oset E, Tegen R and Weise W 1984 Nucl. Phys. A 426456

[13] Gutsche T 1987 Ph. D. Thesis, Florida State University, 249 p. (unpublished)

[14] Gutsche T and Robson D 1989 Phys. Lett. B229 333

[15] Saito K 1984 Prog. of Theor. Phys. V71 775

[16] Nagai S, Miyatsu T and Saito K 2008 Phys. Lett. B666 239

[17] Lüscher M 1981 Nucl. Phys. B180 317

[18] Gell-Mann M and Levy M 1960 Nuovo Cim. 161729

[19] Fetter A I and Waleska J D 1971 Quantum theory of many particle systems (McGraw-Hill, New York) 